\title{
DESENVOLVIMENTO DE SALGADINHOS COM TEORES REDUZIDOS DE GORDURA SATURADA E DE ÁCIDOS GRAXOS TRANS
}

\author{
Vanessa D. CAPRILES ; José A.G. ARÊAS
}

\begin{abstract}
RESUMO
A gordura vegetal hidrogenada, veículo convencional para aromatização de salgadinhos, foi substituída parcial ou totalmente por óleo de canola, gerando um novo salgadinho com $73,8 \%$ de redução da gordura saturada em relação aos salgadinhos disponíveis no mercado e eliminação dos ácidos graxos trans. Foi avaliado o impacto desta substituição sobre as características sensoriais do produto. Ao se substituir totalmente a gordura, a alteração de cor, medida pela variação da somatória dos atributos de cor $\Delta \mathrm{E}$, foi de 2,04 . A substituição da gordura em até $50 \%$ não alterou a textura significativamente $(\mathrm{p}<0,05)$; entretanto, quando esta substituição foi igual ou superior a $75 \%$ houve diferença em relação à amostra padrão. Foi percebida alteração de textura pelos provadores quando a amostra padrão (22\% gordura) foi confrontada com a amostra aromatizada com $22 \%$ de óleo. A amostra padrão apresentou maior aceitabilidade sensorial. Pelos resultados, concluiu-se que as características sensoriais do produto são fracamente afetadas pela substituição da gordura vegetal hidrogenada pelo óleo de canola. Abordagens como esta podem ser utilizadas em escala industrial, gerando produtos diferenciados e contribuindo para diminuição da ingestão dos ácidos graxos saturados e ácidos graxos trans.
\end{abstract}

Palavras-chave: gordura saturada; ácidos graxos trans; salgadinhos; aceitabilidade sensorial; gordura vegetal hidrogenada.

\section{SUMMARY}

DEVELOPMENT OF SNACKS WITH REDUCED SATURATED AND TRANSFATTY ACIDS CONTENTS. The hydrogenated vegetable fat normally used as a vehicle for flavoring snacks, has been partially or totally replaced by rapeseed oil, resulting in a new snack with $73,8 \%$ less saturated fat than regular snacks. This new product doesn't have any trans fatty acids in its composition. The impact of this replacement in sensorial characteristics of this product has been analyzed. In the sample where the fat had been totally replaced the color change was $\Delta \mathrm{E}=2.04$. It has been also noticed that replacement up to $50 \%$ did not cause any significant changes on texture $(\mathrm{p}<0.05)$. However, when this replacement is over $75 \%$, a texture difference was observed as compared to the standard sample. In the sensorial analysis, the standard sample ( $22 \%$ fat) was compared to the one flavored with $22 \%$ rapeseed oil, and the difference in texture was noticed, with the sample flavored with vegetable fat presenting better sensory acceptance. Based on our results, we concluded that the sensorial characteristics of this product were not affected practically after the replacement of regular fat by rapeseed oil, making approaches like this viable to be used on an industrial scale with the purpose to help reducing the intake of saturated and trans fatty acids by the population.

Keywords: saturated fat; trans fatty acids; snacks; sensorial acceptability; hydrogenated vegetable fat.

\section{1 - INTRODUÇÃO}

A extrusão termoplástica é um processo no qual a matéria-prima é forçada a passar através de um molde, em condições de mistura e aquecimento, pressão e fricção que levam à gelatinização do amido e à desnaturação de proteînas. Na saída do equipamento ocorre rápida evaporação da água superaquecida presente, que atravessa o material, reorganizando as moléculas desnaturadas, formando uma rede supramolecular e gerando uma série de produtos estruturados prontos para o consumo ou ingredientes com largo emprego na indústria de alimentos $[4,5,14,15,16,21]$.

Entre estes produtos encontram-se os salgadinhos que são obtidos a partir de milho ou trigo e que apresentam textura altamente apreciada. Para se tornarem mais palatáveis e aceitáveis para o consumo, estes produtos são aromatizados. O aroma e o sabor fixam-se no salga-

Recebido para publicação em 26/04/2004. Aceito para publicação em 18/05/2005 (001329).

Departamento de Nutrição, Faculdade de Saúde Pública, Universidade de São Paulo. Av. Dr. Arnaldo, 715 - CEP: 01246-904. São Paulo-SP, Brasil.

A quem a correspondência deve ser enviada. dinho através de um veículo lipídico, normalmente gordura vegetal hidrogenada. Apesar de ser quimicamente estável, sua utilização se caracteriza como uma desvantagem nutricional, devido aos elevados índices de gordura saturada e de ácidos graxos trans em sua composição [8, 24].

ENIG et al. [11] encontraram cerca de 23,4\% de ácidos graxos trans e TAVELLA et al. [26] 34-39\% de gordura saturada na fração lipídica deste tipo de produto. Sabe-se que a elevada ingestão de ácidos graxos saturados e trans contribui para a hipercolesterolemia. Além disso, os ácidos graxos trans possuem efeitos mais severos que os saturados, pois além de aumentarem os niveis de LDL-colesterol, diminuem simultaneamente os de HDL-colesterol e são compostos que aumentam o risco da ocorrência de câncer [1, 6, 9, 11, 18, 25, 26].

O consumo de ácidos graxos trans da população americana é de aproximadamente $2,6 \%$ do total energético e de 7 a $10 \%$ do total de energia proveniente de lipídios [14, 25]. Ainda não há estudos epidemiológicos deste tipo no Brasil. Porém, como os hábitos alimentares do brasileiro estão cada vez mais próximos do americano, o consumo de ácidos graxos trans também deve estar elevado, uma vez que se observa consumo excessivo de produtos industrializados que apresentam gordura vegetal hidroge- 
nada em sua formulação. [22]

Considerando estes fatores e o aumento da incidência e prevalência de obesidade e doenças cardiovasculares, torna-se uma importante medida de Saúde Pública melhorar a qualidade nutricional dos alimentos industrializados que fazem parte do hábito alimentar da população.

Os objetivos deste trabalho foram reduzir os teores de gordura saturada e de ácidos graxos trans em salgadinhos, através da substituição da gordura vegetal hidrogenada, utilizada para aromatização, por óleo de canola, e averiguar o potencial do novo produto através da avaliação de possivveis alterações sensoriais.

\section{2 - MATERIAL E MÉTODOS}

O protocolo experimental foi aprovado pelo Comitê de Ética em Pesquisa envolvendo seres humanos da Faculdade de Saúde Pública da Universidade de São Paulo. Seguindo a resolução específica do Conselho Nacional de Saúde (nº $196 / 96)$, todos os participantes foram informados detalhadamente sobre os procedimentos utilizados e concordaram em participar de maneira voluntária do estudo, assinando um termo de consentimento informado e de proteção da privacidade.

\section{1 - Produção dos salgadinhos}

A matéria-prima utilizada foi o gritz de milho (Zea mays L.), com umidade ajustada para $16 \%$. Os salgadinhos foram produzidos em extrusora com capacidade industrial (180kg/hora) modelo Inbra 200 INBRAMAQ, Indústria de Máquinas Ltda. (Ribeirão Preto, São Paulo). Foram adotadas as seguintes condições de processamento: camisa cônica e helicoidal; rosca cônica, paralela com quatro entradas e de passo normal; taxa de compressão da rosca de $1: 1$; trafila com seis furos com $3,17 \mathrm{~mm}$ de diâmetro interno; sub- trafila com cento e cinquenta e dois furos com $4 \mathrm{~mm}$ de diâmetro interno; velocidade da rosca em 398rpm; Input de energia de $110 \mathrm{~A}$ em 220V; temperaturas de cada zona do canhão: zona $1-25^{\circ} \mathrm{C}$, zona $2-25^{\circ} \mathrm{C}$ e zona $3-90^{\circ} \mathrm{C}$.

Foram obtidos extrusados com razão de expansão igual a 4,6 no formato cilíndrico. A razão de expansão é o resultado da divisão do diâmetro do salgadinho pelo diâmetro da matriz da extrusora. Para o cálculo, utilizou-se a média do diâmetro de 30 unidades aleatórias da amostra após a saída pela matriz.

A aromatização foi realizada a frio em drageadera do aparelho Erweka modelo AR 400, aspergindo-se metade do veículo lipídico, posteriormente salpicando os ingredientes secos, já misturados, e uma nova aspersão do veículo lipídico. Todas as amostras foram aromatizadas com $1,5 \%$ de cloreto de sódio, glutamato monossódico em quantidade equivalente a $25 \%$ do sal, inosinato dissódico em quantidade equivalente a $5 \%$ do glutamato monossódico, 4\% de aroma de queijo; diferindo apenas na composição e quantidade de veículo lipídico.
Produtos similares disponiveis no mercado apresentam em média 22\% de lípides adicionados para a aromatização. A partir deste valor foram desenvolvidas as amostras.

Na Figura 1 observa-se que as primeiras cinco amostras foram elaboradas de modo que houvesse redução do teor de gordura hidrogenada no veículo lipídico em intervalos de $25 \%$ (100\%, 75\%, 50\%, 25\%, 0\%) e fosse mantida a quantidade de lipides totais usualmente utilizada $(22 \%)$. As últimas quatro amostras foram aromatizadas apenas com óleo de canola, com gradativa redução do teor de lípides adicionado, promovendo, desta forma, não só a melhoria nutricional da fração lipídica, mas também a redução calórica.

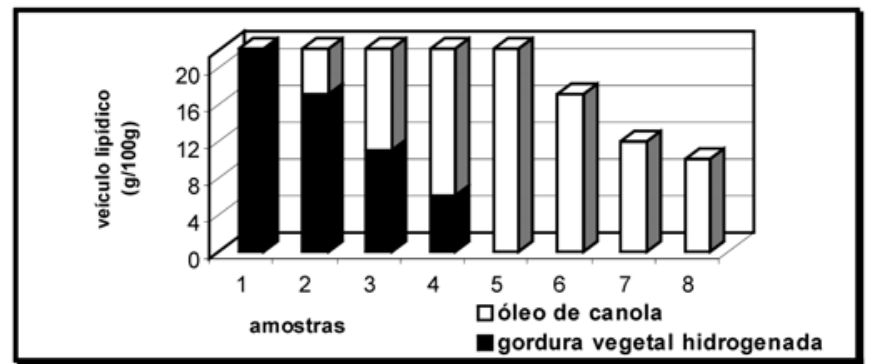

FIGURA 1 - Porcentagens de adição dos componentes do veículo de aromatização aos salgadinhos (considerando a base úmida)

\section{2 - Metodologias analiticas}

\subsection{1 - Perfil de ácidos graxos}

A extração da fração lipídica foi realizada a frio em coluna seca, conforme a metodologia descrita por MARMER \& MAXWELL $[19,20]$, uma alíquota de extrato que continha 50mg de lipídios foi coletada e a derivatização dos ácidos graxos foi realizada conforme o método proposto por HARTMAN \& LAGO [17], sendo que a etapa de saponificação foi realizada através do refluxo dos lípides em condensador com $2 \mathrm{~mL}$ de solução de $\mathrm{NaOH}$ 0,5mol.L' em metanol por 5 minutos. A esterificação foi realizada através da adição de $6 \mathrm{~mL}$ de solução de cloreto de amônio 0,62 mol.L em metanol anidro e ácido sulfúrico (19:1) por 3 minutos. Após a separação da fração de metil ésteres, esta foi lavada com $5 \mathrm{~mL}$ de solução de bicarbonato de sódio saturada para retirar traços do ácido sulfúrico. Os ésteres metílicos de ácidos graxos foram suspensos em hexano e analisados por cromatografia gasosa em coluna capilar (coluna CP-Sil 88 de 50m x $0,25 \mathrm{~mm}$ e $0,2 \mu \mathrm{m}$ de espessura). As condições cromatográficas foram: temperaturas da coluna: inicial $100^{\circ} \mathrm{C}$ por 2 minutos, rampa $5^{\circ} \mathrm{C}$ por minuto (por 28 minutos), final $240^{\circ} \mathrm{C}$; temperatura do detector de ionização de chama $300^{\circ} \mathrm{C}$; temperatura do injetor $270^{\circ} \mathrm{C}$; gás de arraste: hidrogênio com fluxo de $1,5 \mathrm{~mL} /$ minuto, razão de divisão 1: 67. Padrões utilizados: Sigma Aldrich - USA: 189-19 Fatty acid methyl esters mixture, L8404 Linoleic acid methyl ester cis/trans isomers e L6034 Linolenic acid methyl ester, isomer mix. 


\subsection{2 - Cor}

A cor é um atributo tridimensional, constituída pelos parâmetros luminosidade, croma e tom. A luminosidade é a qualidade pela qual se distingue uma cor clara de outra escura; o croma define a "força" ou "pureza" da cor, através deste parâmetro pode-se distinguir uma cor fraca de uma forte e o tom é associado ao nome da cor.

A cor foi verificada através do sistema CIE L*a*b* em aparelho Color Quest XE (Hunter Lab USA). Os valores determinados instrumentalmente são $L^{*}, a^{*}$ e b*. A partir desses valores calculam-se: croma $=\left(\mathrm{a}^{*^{2}+\mathrm{b}^{*^{2}}}\right)^{1 / 2}$ e tom (ângulo $\theta$ ) $=\operatorname{tg} \mathrm{b}^{*} / \mathrm{a}^{*}$. Os valores $\mathrm{L}^{*}$ luminosidade vão de zero (preto) até cem (branco), $a^{*}$ (oposição vermelho - verde): +a (até +100) corresponde ao vermelho, -a (até -80) corresponde ao verde; $b^{*}$ (oposição amarelo - azul): $+\mathrm{b}$ $($ até +70$)$ corresponde ao amarelo e -b (até ${ }^{-100)}$ corresponde ao azul $[7,13]$. A diferença de cor entre duas amostras foi calculada por $\Delta \mathrm{E}$.

$$
\Delta \mathrm{E}=\left[\left(\Delta \mathrm{L}^{*}\right)^{2}+\left(\Delta \mathrm{a}^{*}\right)^{2}+\left(\Delta \mathrm{b}^{*}\right)^{2}\right]^{1 / 2}
$$

sendo: $\Delta \mathrm{L}^{*}=\left(\mathrm{L}_{1}-\mathrm{L}_{2}\right), \quad \Delta \mathrm{a}^{*}=\left(\mathrm{a}_{1}-\mathrm{a}_{1}\right), \quad \Delta \mathrm{b}^{*}=\left(\mathrm{b}_{1}-\mathrm{b}_{2}\right)$; segundo FRANCIS \& CLYDESDALE [13].

\subsection{3 - Textura}

Como parâmetro de textura foi determinada a força necessária para o cisalhamento completo do produto em uma cela do tipo "Warner Bratzler", em aparelho TAXT2i (TA Instruments Inglaterra). O corte foi perpendicular ao comprimento dos salgadinhos e foram utilizados os seguintes parâmetros para análise: velocidade no préteste: $2 \mathrm{~mm} / \mathrm{s}$, velocidade durante o teste: $1 \mathrm{~mm} / \mathrm{s}$, velocidade pós-teste: $5 \mathrm{~mm} / \mathrm{s}$ e distância percorrida: $30 \mathrm{~mm}$ (até o cisalhamento completo).

\subsection{4 - Análise sensorial}

Foi aplicado, através de um painel não treinado constituído por cinqüenta pessoas consumidoras de salgadinhos, um teste afetivo, com escala hedônica de nove pontos $(1$ - desgostei muitíssimo, 5 - indiferente, 9 - gostei muitíssimo) [22] sob luz branca e luz vermelha, ocasião em que há mascaramento da cor. Na análise sensorial a amostra aromatizada com $22 \%$ de óleo foi confrontada com a amostra padrão (salgadinho aromatizado com $22 \%$ de gordura). Cada amostra a ser apresentada para os provadores foi codificada com três dígitos ao acaso e a ordem de apresentação foi aleatória. A análise foi realizada no Laboratório de Análise Sensorial, da Faculdade de Saúde Pública que está equipado com cabines individuais. Inicialmente os salgadinhos foram provados sob luz branca, e em seguida sob luz vermelha.

\section{3 - RESULTADOS E DISCUSSÃO}

\section{1 - Perfil de ácidos graxos}

Através da Tabela 1 percebe-se que o objetivo de melhoria nutricional do perfil de ácidos graxos dos lípides dos salgadinhos foi alcançado, tanto pela substituição total como parcial da gordura vegetal hidrogenada. A substituição progressiva ocasionou redução gradual dos teores de gordura saturada e de ácidos graxos trans, e com a substituição total ocorre a exclusão de ácidos graxos trans no produto.

TABELA 1 - Proporção relativa (\%) dos ácidos graxos dos salgadinhos segundo nível de insaturação e posição geométrica

\begin{tabular}{ccccccccccc}
\hline & \multicolumn{7}{c}{ Amostra } \\
\cline { 2 - 9 } & $22 \% \mathrm{G}$ & $\begin{array}{c}17 \% \mathrm{G} \\
+5 \% \mathrm{O}\end{array}$ & $\begin{array}{c}11 \% \mathrm{G}+ \\
11 \% \mathrm{O}\end{array}$ & $\begin{array}{c}5 \% \mathrm{G}+ \\
17 \% \mathrm{O}\end{array}$ & $22 \% \mathrm{O}$ & $17 \% \mathrm{O}$ & $12 \% \mathrm{O}$ & $10 \% \mathrm{O}$ & Mereado \\
\hline Saturados & 27,5 & 24,4 & 20,0 & 21,8 & 9,2 & 9,1 & 9,1 & 9,4 & 34,0 \\
Trans & 5,0 & 4,1 & 2,9 & 1,5 & 0 & 0 & 0 & 0 & 2,0 \\
Monoinsaturados & 17,8 & 26,0 & 35,0 & 41,0 & 57,2 & 58,4 & 59,0 & 58,2 & 36,0 \\
Polinsaturados & 49,7 & 45,5 & 42,1 & 35,7 & 33,6 & 32,5 & 31,9 & 32,4 & 28,0 \\
\hline G = gordura vegetal hidrogenada $\mathrm{O}=$ óleo de canola & & & & & &
\end{tabular}

Também foi determinado o perfil de ácidos graxos de 14 produtos similares disponíveis no mercado, de diferentes marcas, e constatou-se que, a fração lipídica que varia de 19 a $27 \%$, apresenta em média $34 \%$ de saturados, $2 \%$ de trans, $36 \%$ de monoinsaturados e $28 \%$ de polinsaturados; sendo que o teor de ácidos graxos saturados variou de 13,17 a 49,75\% e o de ácidos graxos trans de 0 a $7,15 \%$.

Ao comparar o perfil de ácidos graxos, observa-se no salgadinho que foi aromatizado com $22 \%$ de óleo de canola uma redução de $66,5 \%$ do conteúdo de ácidos graxos saturados em relação ao produto aromatizado com $22 \%$ de gordura; em relação aos salgadinhos disponíveis no mercado, essa redução é de $73 \%$.

Para determinação dos isômeros cis/trans dos ácidos graxos linoléico e alfa-linolênico foram seguidas as ordens de eluição dos componentes na mesma coluna segundo a descrição da prática recomendada Ch 2a-94 e do método oficial Ce 1f-96 da AOCS para análise de óleos e gorduras comerciais. Os protocolos destas técnicas, assim como o da utilizada neste trabalho não permitiram a separação dos isômeros C18:3 ctc/tcc devido à sobreposição dos picos [2, 3].

A Tabela 2 apresenta a porcentagem de cada ácido graxo com isomeria trans encontrado nos salgadinhos convencionais e nas amostras que apresentam gordura vegetal hidrogenada em sua composição. Observa-se que o ácido graxo trans encontrado em maior quantidade tanto nos produtos desenvolvidos como nos salgadinhos convencionais foi o elaídico (C18:1t); este resultado está de acordo com o observado por CHIARA, SICHIERI \& CARVALHO [10] que avaliaram a composição em ácidos graxos de alguns produtos que possuem gordura vegetal hidrogenada em sua formulação e encontraram este isômero com maior freqüência e em maior quantidade que os demais isômeros trans.

Possivelmente, as gorduras utilizadas na aromatização dos salgadinhos comerciais apresentam maior grau de hidrogenação; processo que consiste na adição de átomos de hidrogênio às duplas ligações dos ácidos graxos insaturados, formando além dos ácidos graxos satura- 
TABELA 2 - Participação percentual de cada ácido graxo com isômeria trans na composição da fração lipídica dos salgadinhos

\begin{tabular}{|c|c|c|c|c|c|}
\hline & \multicolumn{5}{|c|}{ Amostra } \\
\hline & $22 \% \mathrm{G}$ & $17 \% \mathrm{G}+5 \% \mathrm{O}$ & $11 \% \mathrm{G}+11 \% \mathrm{O}$ & $6 \% \mathrm{G}+18 \% \mathrm{O}$ & Mercado \\
\hline C18:19t & 3,68 & 2,95 & 1,99 & 1,15 & 1,15 \\
\hline $\mathrm{C} 18: 29 \mathrm{t} 12 \mathrm{t}$ & 0,21 & 0 & 0,09 & 0 & 0,03 \\
\hline $\mathrm{C} 18: 29 \mathrm{cl} 2 \mathrm{t}$ & 0,49 & 0,40 & 0,35 & 0,23 & 0,35 \\
\hline $\mathrm{C} 18: 29 \mathrm{t} 12 \mathrm{c}$ & 0,40 & 0,40 & 0,28 & 0 & 0,31 \\
\hline $\begin{array}{l}\text { C18:2 t } \\
\text { (Somatório dos isômeros) }\end{array}$ & 1,10 & 0,80 & 0,72 & 0,23 & 0,69 \\
\hline $\mathrm{C} 18: 39 \mathrm{t} 12 \mathrm{cl} 5 \mathrm{t}$ & 0,25 & 0,29 & 0,21 & 0,13 & 0,41 \\
\hline $\mathrm{C} 18: 39 \mathrm{cl} 2 \mathrm{c} 15 \mathrm{t}$ & 0 & 0 & 0 & 0 & 0,56 \\
\hline $\mathrm{Cl} 18: 39 \mathrm{cl} 2 \mathrm{t} 15 \mathrm{c} / 9 \mathrm{t} 12 \mathrm{cl} 5 \mathrm{c}$ & 0 & 0 & 0 & 0 & 0,34 \\
\hline $\begin{array}{l}\text { C18:3 t } \\
\text { (Somatório dos isômeros) }\end{array}$ & 0,25 & 0,29 & 0,21 & 0,13 & 1,31 \\
\hline
\end{tabular}

Desvio Padråo (DP), número de ensaios (n).

$\mathrm{G}=$ gordura vegetal hidrogenada $\mathrm{O}=$ oleo de canola

dos correspondentes, vários isômeros trans [9, 11]. Este fato pode ser constatado através da comparação dos resultados expressos nas Tabelas 1 e 2 que demonstram que os produtos disponiveis no mercado apresentam, em média, maiores teores de ácidos graxos saturados e maior variedade de isômeros trans.

Nos produtos desenvolvidos observa-se maiores teores de isômeros trans do ácido linoléico, que é o ácido graxo presente em maiores concentrações na gordura utilizada.

Para a aromatização dos salgadinhos desenvolvidos neste trabalho foi utilizada uma gordura hidrogenada que possui altos teores de ácidos graxos insaturados. Com a utilização desta gordura que não necessita aquecimento para sua fusão, foram evitadas possiveis alterações na composição em ácidos graxos em função do aquecimento.

\section{2 - Cor}

A Tabela 3 apresenta as médias e os intervalos de variação das amostras em cada parâmetro de cor. Também foi calculado o $\Delta \mathrm{E}$, que é um indicador da diferença de cor entre duas amostras [13] (Tabela 4).

TABELA 3 - Parâmetros de cor dos salgadinhos em função da composição do veículo lipídico utilizado na aromatização

\begin{tabular}{ccccccc}
\hline & \multicolumn{6}{c}{ Amostra } \\
\cline { 2 - 7 } & \multicolumn{6}{c}{ Cor (média \pm DP) } \\
& $\mathrm{n}$ & $\mathrm{L}^{*}$ & $\mathrm{a}^{*}$ & $\mathrm{~b}^{*}$ & Croma & Tom \\
\hline $22 \% \mathrm{G}$ & 3 & $76,8 \pm 0,38$ & $4,72 \pm 0,11$ & $38,2 \pm 0,20$ & $38,0 \pm 0,21$ & $82,9 \pm 0,13$ \\
$17 \% \mathrm{G}+5 \% \mathrm{O}$ & 3 & $77,5 \pm 0,4$ & $3,71 \pm 0,09$ & $36,4 \pm 0,05$ & $37,4 \pm 1,8$ & $83,4 \pm 0,85$ \\
$11 \% \mathrm{G}+11 \% \mathrm{O}$ & 3 & $77,9 \pm 0,21$ & $5,01 \pm 0,49$ & $38,4 \pm 1,2$ & $36,7 \pm 0,26$ & $83,9 \pm 0,86$ \\
$6 \% \mathrm{G}+18 \% \mathrm{O}$ & 3 & $76,6 \pm 0,52$ & $5,45 \pm 0,16$ & $39,1 \pm 0,12$ & $36,6 \pm 0,18$ & $84,3 \pm 0,09$ \\
$22 \% \mathrm{O}$ & 3 & $77,3 \pm 0,50$ & $3,64 \pm 0,07$ & $36,5 \pm 0,26$ & $38,1 \pm 0,96$ & $83,4 \pm 0,84$ \\
$17 \% \mathrm{O}$ & 3 & $77,5 \pm 0,58$ & $4,37 \pm 0,49$ & $38,5 \pm 1,28$ & $36,7 \pm 0,13$ & $84,2 \pm 0,11$ \\
$12 \% \mathrm{O}$ & 3 & $77,1 \pm 0,04$ & $4,28 \pm 0,75$ & $37,6 \pm 1,85$ & $36,6 \pm 0,06$ & $84,2 \pm 0,13$ \\
$10 \% \mathrm{O}$ & 3 & $76,6 \pm 0,87$ & $3,86 \pm 0,65$ & $36,5 \pm 2,01$ & $36,8 \pm 0,38$ & $83,8 \pm 0,55$ \\
\hline
\end{tabular}

Desvio Padrào (DP), número de ensaios (n).

$\mathrm{G}=$ gordura vegetal hidrogernda $\mathrm{O}=$ óleo de canola

Os resultados obtidos para cada parâmetro de cor foram interpretados em dois blocos, de acordo com a representação das amostras na Figura 1, considerando-se a redução da gordura vegetal hidrogenada na mistura lipídica (amostras $1,2,3,4,5)$ e considerando-se a redução da quantidade total de lipides utilizados na aromatização (amostras 5,6,7,8).

\subsection{1 - Redução da gordura vegetal hidrogenada no veículo lipídico}

Os resultados expressos nas Tabelas 3 e 4 demonstram não haver dependência nos parâmetros de cor dos salgadinhos em função da redução gradual da gordura vegetal hidrogenada na mistura lipídica utilizada para aromatizar o produto.

TABELA 4 - Variação dos parâmetros de cor dos salgadinhos em função da substituição gradual da gordura vegetal hidrogenada utilizada na aromatização (em relação a amostra $22 \% \mathrm{G}$ )

\begin{tabular}{ccccc}
\hline \multirow{2}{*}{ Amostra } & $\Delta \mathrm{L}^{*}$ & \multicolumn{4}{c}{ Variação dos parâmetros de cor } \\
& $-0,76$ & 1,01 & $\Delta \mathrm{a}^{*}$ & $\Delta \mathrm{E}$ \\
\hline $17 \% \mathrm{G}+5 \% \mathrm{O}$ & $-1,11$ & $-0,29$ & $-0,2$ & 2,2 \\
$11 \% \mathrm{G}+11 \% \mathrm{O}$ & 0,15 & $-0,73$ & $-0,9$ & 1,16 \\
$6 \% \mathrm{G}+18 \% \mathrm{O}$ & $-0,49$ & 1,08 & 1,66 & 2,17 \\
$22 \% \mathrm{O}$ & &
\end{tabular}

$\mathrm{G}=$ gordura vegetal hidrogenada $\mathrm{O}=$ óleo de canola

\subsection{2 - Redução da quantidade de óleo utilizada na aromatização}

Através dos resultados expressos nas Tabelas 3 e 5 nota-se que não houve diferença de luminosidade e tom entre as amostras aromatizadas com 17, 12 e 10\% de óleo de canola em relação a amostra que foi aromatizada com $22 \%$ deste óleo. As amostras que foram aromatizadas com 17,12 e $10 \%$ de óleo de canola não apresentaram diferenças entre si em nenhum parâmetro de cor.

A regressão linear (Figura 2) desses resultados demonstra que a redução da quantidade de óleo de canola utilizado para aromatizar ocasiona uma alteração direta-

TABELA 5 - Variação dos parâmetros de cor dos salgadinhos em função da redução do teor de óleo de canola utilizado na aromatização (em relação a amostra 22\%O)

\begin{tabular}{ccccc}
\hline \multirow{2}{*}{ Amostra } & $\Delta \mathrm{L}^{*}$ & \multicolumn{4}{c}{ Variação dos parâmetros de cor } \\
& $-0,18$ & $-0,73$ & $-1,95$ & $\Delta \mathrm{E}$ \\
\hline $17 \% \mathrm{O}$ & 0,15 & $-0,64$ & $-1,08$ & 2,09 \\
$12 \% \mathrm{O}$ & 0,64 & $-0,22$ & 0,06 & 0,68 \\
$10 \% \mathrm{O}$ & & &
\end{tabular}

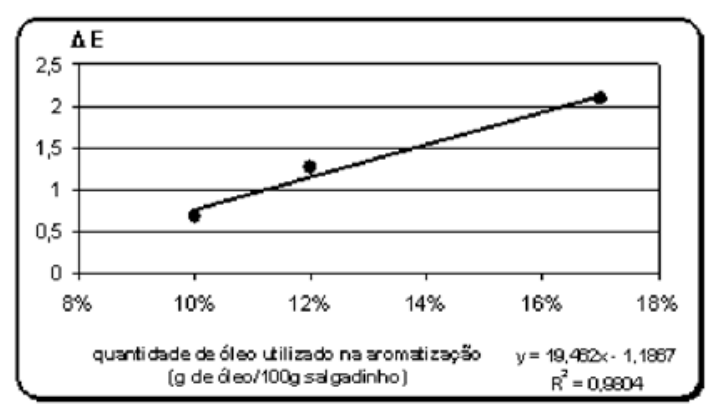

FIGURA 2 - Alteração de cor dos salgadinhos em função da diminuição do teor de óleo de canola utilizado na aromatização 
mente proporcional $\left(\mathrm{R}^{2}=0,98\right)$ no valor de $\Delta \mathrm{E}$. Quanto menor a quantidade de óleo maior a redução do $\Delta \mathrm{E}$ em relação a cor do salgadinho aromatizado com $22 \%$ de óleo de canola.

Com base nestas observações é possivel afirmar que foram identificadas instrumentalmente pequenas variações nos parâmetros de cor das amostras, entretanto, visualmente estas alterações não foram perceptiveis.

\section{3 - Textura}

Os resultados obtidos para a avaliação de textura foram interpretados através de análise de variância (ANOVA). Foi feita análise estatística comparativa de médias através do teste de Tukey, duas a duas na ordem em que foram apresentadas na Figura 1, adotando-se um nivel de confiança de 95\% [12,23].

TABELA 6 - Médias descritivas da textura dos salgadinhos em função da composição do veículo lipídico utilizado na aromatização

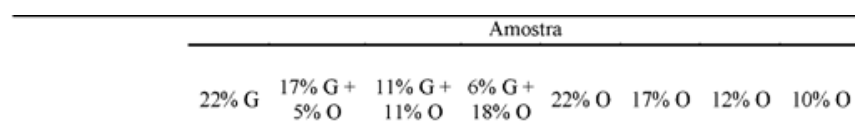

\begin{tabular}{|c|c|c|c|c|c|c|c|c|}
\hline $\begin{array}{c}\text { Força de } \\
\text { cisalhamento }(\mathrm{N})\end{array}$ & $53,7^{\mathrm{A}}$ & $53,5^{a}$ & $45,9^{\text {tb }}$ & $39^{\circ}$ & $62,8^{c}$ & $42,3^{\mathrm{b}}$ & $41,8^{b}$ & $41,9^{h}$ \\
\hline $\mathrm{N}$ & 15 & 15 & 15 & 15 & 15 & 15 & 15 & 15 \\
\hline
\end{tabular}

Os resultados de textura também foram analisados em dois blocos.

\subsection{1- Redução da gordura vegetal hidrogenada no veículo lipídico}

Observa-se na Tabela 6 que, a substituição da gordura vegetal hidrogenada em até 50\% (11\% G + 11\% O) não altera significativamente a textura. Entretanto, quando esta substituição é igual a $75 \%(6 \% \mathrm{G}+18 \% \mathrm{O})$ há diferença de textura em relação às amostras aromatizadas com $100 \%$ (22\% G) e $75 \%$ (17\% G + 5\% O) de gordura na mistura lipídica.

A regressão linear (Figura 3) desses resultados demonstra que a redução progressiva em $25 \%$ do teor de gordura na mistura lipídica utilizada para a aromatização dos snacks ocasiona proporcional redução da força de cisalhamento $\left(R^{2}=0,91\right)$. Este comportamento se deve à diminuição proporcional dos teores de ácidos saturados e trans, que são sólidos à temperatura ambiente e, por isso, podem ocasionar maior força necessária para o cisalhamento.

\subsection{2 - Redução da quantidade de óleo utilizada na aromatização}

O salgadinho aromatizado com $22 \%$ de óleo destacou-se dos demais em relação à textura. Salgadinhos aromatizados com as quantidades 17,12 e $10 \%$ de óleo de canola não apresentaram diferença estatisticamente significativa em relação à textura $(\mathrm{p}<0,05)$.

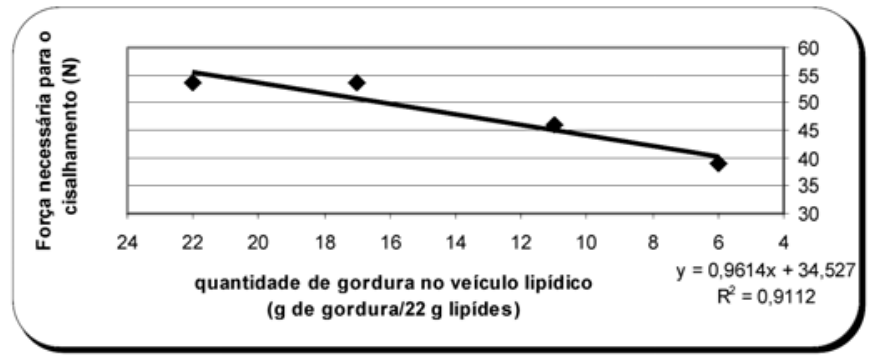

FIGURA 3 - Influência da redução progressiva da quantidade de gordura vegetal hidrogenada no veículo lipídico sobre a força necessária para o cisalhamento dos salgadinhos

Através dos resultados de textura observa-se que não houve alteração de textura dependente da redução do teor de óleo. Nota-se uma queda brusca na força de cisalhamento quando houve redução de $22 \%$ para $17 \%$ de óleo na aromatização do produto, porém, este valor praticamente se mantém quando é utilizado 17,12 e $10 \%$ de óleo.

\section{4 - Análise sensorial}

Os resultados obtidos na análise sensorial foram avaliados através de análise de variância (ANOVA). Foi feita análise estatística comparativa das notas médias obtidas pelas amostras através do teste de Tukey, adotandose um nivel de confiança de 95\% [12, 23].

Através dos resultados expressos na Tabela 7 notase que, os dois produtos foram aceitos tanto sob luz branca como sob luz vermelha, já que as notas médias encontram-se acima de 6 (gostei ligeiramente) e mais da metade dos provadores atribuíram notas acima do ponto de corte, notas $\geq 7$. Os valores de moda demonstraram que a maior proporção de provadores atribuíram nota igual a 7 para o salgadinho com óleo analisado sob luz vermelha e nota 8 quando analisado sob luz branca. Foi atribuída nota 8 para o salgadinho aromatizado com gordura nas duas circunstâncias.

TABELA 7 - Aceitabilidade sensorial dos salgadinhos aromatizados com $22 \%$ de gordura vegetal hidrogenada e $22 \%$ de oleo de canola

\begin{tabular}{ccccc}
\hline Amostra & $\mathrm{n}$ & Média das notas & Aceitação (\%) & Moda \\
\hline & \multicolumn{5}{c}{ Luz branca } \\
$22 \%$ de óleo & 50 & $6,88^{\mathrm{ab}}$ & 68 & 8 \\
$22 \%$ de gordura & 50 & $7,5^{\mathrm{c}}$ & 86 & 8 \\
\hline & \multicolumn{5}{c}{ Luz vermelha } \\
$22 \%$ de óleo & 50 & $6,52^{\mathrm{a}}$ & 56 & 7 \\
$22 \%$ de gordura & 50 & $7,32^{\mathrm{bc}}$ & 84 & 8 \\
\hline Desvio padråo (DP), número de provadores (n), aceitação refere-se à porcentagem de notas $\geq 7$, mesmas letras
\end{tabular}

significam médias estatisticamente iguais (Teste de Tukey $=0,56$ a um nivel de significância de $95 \%$ ).

Não houve diferença estatisticamente significativa entre as notas atribuidas ao mesmo produto sob luz branca ou vermelha. Entretanto, houve diferença significativa entre os produtos demonstrando que a diferença entre estes salgadinhos é percebida pelos provadores, e que o salgadinho padrão ( $22 \% \mathrm{G})$ apresenta maior aceitabilidade, o que é comprovado pela maior média das notas e pe- 
la quantidade superior de notas acima do ponto de corte, tanto sob luz branca como sob luz vermelha.

Os salgadinhos confrontados na análise sensorial apresentaram diferenças de cor e de textura. Acredita-se que a diferença de aceitabilidade deva-se à textura, pois além da mensuração instrumental, no formulário para avaliação sensorial havia um campo opcional para que o provador se manifestasse. Dentre as manifestações espontâneas relacionadas à textura, 53\% eram de reprovação do salgadinho aromatizado com óleo, tanto sob luz branca, como vermelha. Quando a análise sensorial é realizada com mascaramento da cor, o degustador se atem a outras características, pois a diferença de cor é neutralizada. Nesta situação, a única diferença entre os produtos foi a textura. Na luz vermelha, a influência negativa da textura na aceitabilidade intensificou-se. A aprovação da textura deste produto caiu de $12 \%$ para $0 \%$, justificando, desta forma, as pequenas reduções no valor da moda, da nota média e da porcentagem de notas acima do ponto de corte atribuídas a amostra aromatizada com óleo, enquanto que a aromatizada com gordura manteve a aceitabilidade.

Neste trabalho, pôde-se observar a viabilidade nutricional e comercial da substituição da gordura vegetal hidrogenada por óleo de canola para a aromatização de salgadinhos. Com isso, pôde-se produzir um novo produto com alta aceitabilidade sensorial e com reduzidos teores de gordura saturada e isento de ácidos graxos trans.

Observa-se uma tendência mundial em relação à obrigatoriedade da declaração do conteúdo de ácidos graxos trans na rotulagem nutricional. Desta forma, as indústrias de alimentos deverão se esforçar para diminuir os niveis ou excluir esta substância de seus produtos. Neste sentido, abordagens como a utilizada neste trabalho deverão ser empregadas em escala industrial, promovendo a diferenciação do produto no mercado e também contribuindo para a diminuição da ingestão destes ácidos graxos. Os dados sugerem que uma melhoria tecnológica em relação à textura pode aumentar a aceitabilidade sensorial deste novo salgadinho.

\section{4 - CONCLUSÕES}

Através da substituição parcial ou total da gordura vegetal hidrogenada pelo óleo de canola, para compor o veículo de aromatização de salgadinhos, foi possível reduzir os teores de gordura saturada e de ácidos graxos trans. E com a substituição total ocorreu a exclusão de ácidos graxos trans do produto.

A cor, textura e aceitabilidade sensorial foram fracamente afetadas pela substituição da gordura vegetal hidrogenada pelo óleo de canola, demonstrando assim, a viabilidade comercial deste novo produto.

\section{5 - REFERÊNCIAS BIBLIOGRÁFICAS}

[1] ALLISON, D.B.; EGAN, K.S.; BARRAY, L.M.; CAUGHMAN, C.; INFANTE, M.; HEIMBACK, J.T. Estimated intakes of trans fatty and other fatty acids in the US population. J. Am. Diet. Assoc. v. 99, p.166-174, 1999.

[2] AOCS. Trans unsaturated fatty acids by capillary column gas chromatography. AOCS Recommended Practice $\mathrm{Ch}$ $2 a-94$.

[3] AOCS. Determination of cis and trans fatty acids in hydrogenated and refined oils and fats by capillary GLC. AOCs Official Method Ce 1f-96.

[4] ARÊAS, J.A.G. Extrusion of food proteins. Crit. Rev. Food Sci. Nutr.v.32, p.365-392, 1992.

[5] AREAAS, J.A.G. Interações moleculares do amido durante o processo de extrusão. Bol. SBCTA. v.30, n.1, p28-30, 1996.

[6] ASCHERIO, A.; WILLETT, W. Health effects of trans fatty acids. Am. J. Clin. Nutr. v.66: 1006-1010, 1997.

[7] BEERING, M. Techniques for Measuring Color. Metal Finishing. v.97, n.5, p. 552-557, 1999.

[8] BLOCK J.M.; BARRERA ARELLANO, D. Produtos hidrogenados no Brasil: isômeros trans, caracteristicas físicoquímicas e composição em ácidos graxos. Arch. Latinoam. Nutr.v.44, n.4, p. 281-285, 1994.

[9] CHAIR, E.B.F.; KRIS ETHERTON, P.M.; KRITCHVSKY, D.; LICHTENSTEIN, A.H. Position paper on trans fatty acids. Am. J. Clin. Nutr. v.63, n.5, p.663- 670, 1996.

[10] CHIARA, V.L.; SICHIERI, R.; CARVALHO, T.D.S.F.D. Teores de ácidos graxos trans de alguns alimentos consumidos no Rio de Janeiro. Rev. Nutr. v.16, n.2, p.227233,2003.

[11] ENIG, M.G.; PALLANSCH, L.A.; SAMPUGNA, J.; KEENEY, M. Fatty Acid Composition of the Fat in Select Food Items with Emphasis on trans Components. JAOCS. v.60, n.10, p. 1788-1795, 1983.

[12] FISHER, L.D.; BELLE, G.V. Biostatistics: A methodology for the Health Sciences. $8^{\mathrm{a}}$ ed. Estados Unidos da América: John Wiley \& Sons, Inc; 1993.

[13] FRANCIS, F.J.; CLYDESDALE, F.M. Food Colorimetry: Theory and Applications. The AVI Publishong Co., Inc., 1975 In: FERREIRA, V.L.P., Principios e aplicações da colorimetria em alimentos. Campinas: ITAL, 1981.

[14] GOMEZ, M.H.; AGUILERA, J.M. Changes in the starch fraction during extrusion cooking of corn. J. Food Sci. v.48, n.2, p.378-381. 1983.

[15] HARPER, J.M. Extrusion texturization of foods. Food Technol. v.40, n.3, p.70-82, 1986.

[16] HARPER, J.M. Food Extruders and Their Apllications. In: MERCIER, C.; LINKO, P.; HARPER, J.M. Extrusion Cooking. St.Paul, Minnesota E.U.A.: American Association of Cereal Chemists; 1989.

[17] HARTMAN, L.; LAGO, R.C.A. Rapid Preparation of fatty acid methyl esters from lipids. Lab. Pract. v.22, p. 475$476,1973$.

[18] JUDD, J.T.; CKEVIDENCE, B.A.; MUESING, R.A.; WITTES, J.; SUNKIN, M.E.; PODCZASY JJ. Dietary trans fatty acids: effects on plasma lipids and lipoproteins of healthy men and women. Am. J. Clin. Nutr. v.59, p. 861$868,1994$.

[19] MARMER, W.N.; MAXWELL, R.J. Dry Column Method for 
the Quantitative Extraction and Simultaneous Class Separation of Lipids from Muscle Tissue. Lipids. v.5, p.365-71, 1981

[20] MAXWELL, R.J; MARMER, W.N.; ZUBILLAGA, M.P.; DALICKAS, G.A. Determination of Total Fat in Meat Products by a Rapid, Dry Column Method. J. Assoc. off. Anal. Chem. v.3, p. 600-603, 1980;.

[21] MITCHELL, J.R.; AREAS, J.A.G. Strutural in biopolymers during extrusion. In: KOKINI, J.L.; HO, C.T.; KARWE, M.V. Food Extrusion Science and Technology. New York: Marcel Dekker; 1992. p.345360.

[22] MONDINI, L.; MONTEIRO, C.A. Mudanças no padrão da alimentação. In: Monteiro CA. Velhos e novos males da saúde no Brasil: a evolução do país e de suas doenças. .São Paulo: Hucitec; 1995. p.79-89.

[23] MORAES, M.A.C. Métodos de avaliação sensorial de alimentos. $8^{a}$ ed. Campinas: Editora da Universidade Estadual de Campinas; 1993.

[24] SOARES, L.M.V.; FRANCO, M.R.B. Niveis de trans isômeros e composição de ácidos graxos em margarinas nacionais e produtos hidrogenados semelhantes. Ciên.
Tecnol. Aliment. v.10, n.1, p.57-71, 1990.

[25] SUBBAIAH, P.V.; SUBRAMANIAN, V.S.; LIU, M. Trans unsaturated fatty acids inhibit lecithin: cholesterol acyltransferese and alter its positional specificity. Journal of Lipid Research. v.39, p.1438-1447, 1998.

[26] TAVella, M.; PETERson, G.; ESPECHE, M.; CAVAllero, E.; Cipolla, L.; PEREGO, L.; CABALLERO, B. Trans fatty acid content of a selection of foods in Argentina. Food Chemistry. v.69, p.209-213, 2000.

\section{6 - AGRADECIMENTOS}

À Geni Sampaio, Rosana Aparecida Manólio Soares, Rosa Nilda Chávez - Jauregui, José Perreira, Eric Cassidori Coli, Prof ${ }^{\mathrm{a}}$ Dr $^{\mathrm{a}}$ Elizabeth Pinto Machado e $\operatorname{Prof}^{\mathrm{a}}$ $\mathrm{Dr}^{\mathrm{a}}$ Elizabeth E.A.F.S. Torres, aos estudantes de estatística Alonso Massao Yamaguchi e Adriana Silva e Moura e aos voluntários que participaram da sessão de análise sensorial. Às indústrias: INBRAMAQ, NORSAL, AJINOMOTO, RAI INGREDIENTS, CARGIL, MAEDA. À FAPESP pelo auxílio financeiro e bolsa concedidos. 